ARTICLE OPEN

\title{
Entropy contributions to phase stability in binary random solid solutions
}

\author{
Anus Manzoor ${ }^{1}$, Shubham Pandey ${ }^{2}$, Debajit Chakraborty ${ }^{1}$, Simon R. Phillpot ${ }^{2}$ and Dilpuneet S. Aidhy ${ }^{1}$
}

High entropy alloys contain multiple elements in large proportions that make them prone to phase separation. These alloys generally have shallow enthalpy of mixing which makes the entropy contributions of similar magnitude. As a result, the phase stability of these alloys is equally dependent on enthalpy and entropy of mixing and understanding the individual contribution of thermodynamic properties is critical. In the overall vision of designing high entropy alloys, in this work, using density functional theory calculations, we elucidate the contributions of various entropies, i.e., vibrational, electronic and configurational towards the phase stability of binary alloys. We show that the contribution of electronic entropy is very small compared to the vibrational and configurational entropies, and does not play a significant role in the phase stability of alloys. The configurational and vibrational entropies can either destabilize or can collectively contribute to stabilize the solid solutions. As a result, even those systems that have negative mixing enthalpy can show phase instability, revealed as a miscibility gap; conversely, systems with positive mixing enthalpy can be phase stable due to entropic contributions. We suggest that including entropic contributions are critical in the development of theoretical framework for the computational prediction of stable, single-phase high entropy alloys that have shallow mixing enthalpies, unlike ordered intermetallics.

npj Computational Materials (2018)4:47; doi:10.1038/s41524-018-0102-y

\section{INTRODUCTION}

The phase stability of an alloy is guided by Gibbs free energy $\left(\Delta G_{\text {mix }}\right)$ that comprises of enthalpy $\left(\Delta H_{\text {mix }}\right)$ and entropy $\left(\Delta S_{\text {mix }}\right)$ of mixing. Conventionally, $\Delta H_{\text {mix }}$ has been perceived as the dominant quantity, where a strongly negative $\Delta H_{\text {mix }}$ indicates formation of a stable solid solution and a positive $\Delta H_{\text {mix }}$ indicates unmixing. Theoretical advancements in alloy theory over the past three decades have enabled computational prediction of correct ordered phases and ground-state crystal structures. ${ }^{1-9}$ These methods have relied on analyzing $\Delta H_{\text {mix }}$ in identifying stable metal alloys. In the same vein, $\Delta S_{\text {mix }}$ contributions have also been included in the calculations, albeit selectively; including $\Delta S_{\text {mix }}$ has shown to improve the accuracy of the predicted thermodynamic quantities, such as the order-disorder phase transformation temperature and miscibility gap temperature. ${ }^{3,4,10-15}$ The contribution of $\Delta S_{\text {mix }}$ has often been considered less important, largely due to its relatively smaller size compared to $\Delta H_{\text {mix }}$ particularly in the prediction of ordered structures. However, in the past decade, with the development of high entropy alloys (HEAs), ${ }^{16,17}$ it is gradually becoming evident that $\Delta S_{\text {mix }}$ might be equally important in the phase stability predictions. HEAs are materials that contain random distribution of multiple elements, conventionally five or more, in approximately equal concentrations in a single-phase solid solution. Despite the random distribution of atoms, HEAs are structurally ordered forming simple face centered cubic (fcc) and body centered cubic (bcc) crystal structures. ${ }^{16,18}$ The stability of a single-phase HEA is due to a relatively shallow negative $\Delta H_{\text {mix }}{ }^{19,20}$ among the constituting elements which prevents phase separation and formation of a second sub-phase. The shallow $\Delta H_{\text {mix }}$ in HEAs is in contrast to a deep $\Delta H_{\text {mix }}$ in the ordered alloys or intermetallics. In addition, due to the random distribution of multiple elements, the configurational entropy $\left(\Delta S_{\text {conf }}\right)$ is higher in HEAs than in the ordered alloys, which further contributes to the HEA phase stability. Thus, due to a shallow $\Delta H_{\text {mix }}$ and an unusually high $\Delta S_{\text {conf }}$, the magnitude of entropy contributions can be potentially equal to that of enthalpy in HEAs, thereby making entropy an equally important thermodynamic quantity in phase stability.

High $\Delta S_{\text {conf }}$ has been perceived to be the main reason for the stability of a single phase solid solution in HEAs. ${ }^{16,21}$ It has been argued that increasing the number of elements significantly increases the configurational entropy, and leads to phase stability. However, more recently, it has been shown that the contributions of configurational entropy might only be a part of a bigger picture, and it may not be the only quantity to be considered while explaining the stability of HEAs. ${ }^{22-24}$ For example, using the Hume-Rothery rules as a guide, Otto et al. ${ }^{22}$ experimentally showed that the configurational entropy may not be enough to explain the stability of NiCrCoMnFe HEAs. In a series of experiments, they systematically replaced one element at a time in $\mathrm{NiCrCoMnFe} \mathrm{single} \mathrm{phase-fcc} \mathrm{HEA} \mathrm{with} \mathrm{a} \mathrm{similar} \mathrm{element} \mathrm{(e.g.,}$ $\mathrm{Ni}$ with $\mathrm{Cu}$, Co with Ti). While this strategy should not significantly change the configurational entropy contribution, none of the substitutions led to the formation of a single-phase solid solution HEA. The authors concluded that along with the enthalpy contributions, the non-configurational entropy contributions might be equally important towards the phase stability of HEAs.

Among the various non-configurational entropies, vibrational entropy generally has a significant contribution to $\Delta G_{\text {mix }}$. While

\footnotetext{
'Department of Mechanical Engineering, University of Wyoming, Laramie, WY 82071, USA and ${ }^{2}$ Department of Materials Science and Engineering, University of Florida, Gainesville, FL 32611, USA

Correspondence: Dilpuneet S. Aidhy (daidhy@uwyo.edu)
}

Received: 2 August 2017 Revised: 26 July 2018 Accepted: 1 August 2018

Published online: 22 August 2018 
Table 1. Four possible cases of miscibility gap based on $\Delta H_{\text {mix }}(\checkmark$ and $\boldsymbol{x}$ indicate presence and absence of miscibility gap, respectively)

\begin{tabular}{lll}
\hline Case & & Miscibility gap \\
\hline 1 & $\Delta H_{\text {mix }} \leq 0$ & $\times$ \\
2 & $\Delta H_{\text {mix }} \geq 0$ & $\checkmark$ \\
3 & $\Delta H_{\text {mix }} \leq 0$ & $\checkmark$ \\
4 & $\Delta H_{\text {mix }} \geq 0$ & $x$
\end{tabular}

$\Delta S_{\text {conf }}$ always contributes towards the stability by lowering $\Delta G_{\text {mix }}$ the contribution of $\Delta \mathrm{S}_{\text {vib }}$ is not so straightforward; $\Delta S_{\text {vib }}$ of a solid solution could be positive or negative which may stabilize or destabilize the system. As a result, for those solid solutions that have a narrow range of $\Delta H_{\text {mix }}$ (say, -50 to $+50 \mathrm{meV} /$ atom), the vibrational entropy can have a significant impact on their stability. Its favorable contribution can even stabilize solid solutions that have positive $\Delta H_{\text {mix }}$, or it can even destabilize those that have a negative $\Delta H_{\text {mix }}$. Similarly, the vibrational entropy can either enhance or counteract the stability provided by the configurational entropy. Since, it is still not possible to predict with certainty which combination of elements will lead to formation of a single phase solid solution, ${ }^{19}$ understanding the individual contributions of the key thermodynamic quantities (enthalpy and entropy) and relating them to the existing phase diagrams is crucial. In addition, understanding the intricate correlation of these quantities towards the overall phase stability is vital for the development of a robust thermodynamic model for multicomponent systems.

To elucidate the impact of the delicate balance between the entropy and enthalpy on the phase diagrams in the simplest possible systems, we focus on binary solid solutions in which $\Delta H_{\text {mix }}$ is small and of either sign. In such cases phase stability can be equally controlled by $\Delta H_{\text {mix }}$ and $\Delta S_{\text {mix }}$. We do not consider cases that have a strong negative or positive $\Delta H_{\text {mix }}$, because they will form ordered alloys or will phase separate, respectively, and the effect of entropy will not be important. In the weakly negative $\Delta H_{\text {mix }}$ solid solutions, formation of a random solid solution has a higher probability than an ordered structure. In contrast, in positive $\Delta H_{\text {mix }}$ solid solutions, the phase instability is likely to be evidenced in the form of a two-phase mixture or a miscibility gap. Due to weak negative or positive $\Delta H_{\text {mix }}$, the entropy contributions could become comparable, which could impact the phase stability. Here, by taking examples of several binary phase diagrams, we show that the appearance of a miscibility gap is controlled by the intricate interplay of the enthalpy of mixing, and of the configurational and vibrational entropies. To illustrate, Table 1 lists four possible cases that can lead to the presence or absence of a miscibility gap. Simply based on $\Delta H_{\text {mix }}$, for cases 1 and 2, where $\Delta H_{\text {mix }} \leq 0$ and $\Delta H_{\text {mix }} \geq 0$ respectively, the absence $(x)$ and presence $(\boldsymbol{\checkmark})$ of a miscibility gap is intuitively expected. However, when $\Delta S_{\text {mix }}$ contributions are included, the trends can reverse. As shown by case 3 , even when $\Delta H_{\text {mix }} \leq 0$, due to the overall negative contribution of $\Delta S_{\text {mix }}$, the miscibility gap can appear. Similarly, due to the positive contribution of $\Delta S_{\text {mix }}$, the miscibility gap can disappear even when $\Delta H_{\text {mix }} \geq 0$, as shown in case 4 . In this paper, by performing density functional theory (DFT) calculations on multiple binary solid solutions, we provide an example for each one of the four cases, and elucidate a profound impact of $\Delta S_{\text {mix }}$ on the overall shape of a phase diagram. We also disentangle the individual effects of electronic, vibrational and configurational entropy contributions to the presence and absence of a miscibility gap. Our calculations show that by including the three entropies (in particular vibrational and configurational), the behavior of the miscibility gap can be correctly predicted. In addition, the transformation temperature, i.e., the temperature at which the miscibility gap ends for a given composition, can also be
Table 2. Comparison of order-disorder phase-transition temperatures (K) with literature for $\mathrm{Cu}-\mathrm{Au}, \mathrm{Ni}-\mathrm{Fe}$, and $\mathrm{Ni}-\mathrm{Pt}$

\begin{tabular}{llll}
\hline Systems & $\mathrm{Cu}-\mathrm{Au}$ & $\mathrm{Ni}-\mathrm{Fe}$ & $\mathrm{Ni}-\mathrm{Pt}$ \\
\hline Exp. literature & $683^{26}$ & $618^{27}$ & $900^{28}$ \\
Comp. literature & $560^{4}$ & Not available & $755^{29}$ \\
This work & 700 & 700 & 730 \\
\hline
\end{tabular}

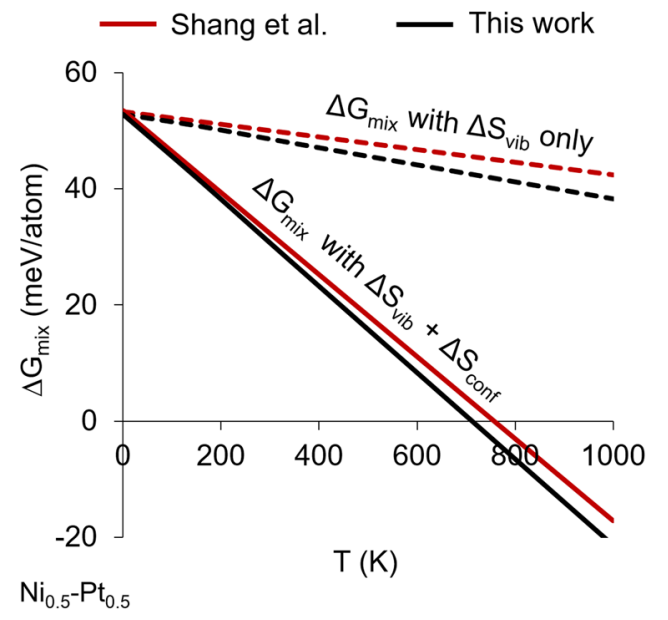

Fig. $1 \Delta G_{\text {mix }}$ vs $T$ comparison with Shang et al. ${ }^{29}$ in $\mathrm{Ni}_{0.5}-\mathrm{Pt}_{0.5}$ system. A difference of only $25 \mathrm{~K}$ in the order-disorder transformation temperature is found

accurately predicted. The understanding developed in this work is expected to contribute to the computational prediction of phase stability of multicomponent HEAs that have narrow $\Delta H_{\text {mix }}$ where entropy contributions are critical.

\section{RESULTS}

The phase stability of the alloys is illustrated using the standard convex-hull approach. In this approach, the alloy is phase-stable if its free energy lies below the tie line formed by connecting the free energies of the two adjacent phases. ${ }^{25}$ In contrast, the alloy phase separates into two phases if its free energy lies above the tie line. We use $\Delta G_{\text {mix }}$ vs $T$ plots to predict the miscibility-gap temperature, i.e., the temperature at which the miscibility gap ends (or the temperature at which a single phase random solid solution begins to be stable). We also show the individual plots for $-T \Delta S_{\text {elec }}-T \Delta S_{\text {vib }}$ and $-T \Delta S_{\text {conf }}$ vs $T$, to disentangle the electronic, vibrational, and configurational entropy contributions. Finally, the ratios of the three entropies contributing towards the phase stabilities of each of the binary alloys are presented. Their ratio indicates the contribution of each towards the phase stability. In this work, examples from fcc and bcc solid solutions are considered; the fcc binary alloys considered are $\mathrm{Ag}-\mathrm{Au}, \mathrm{Pt}-\mathrm{Ru}$, $\mathrm{Ni}-\mathrm{Cu}, \mathrm{Ni}-\mathrm{Rh}, \mathrm{Pd}-\mathrm{Ag}$ and $\mathrm{Ni}-\mathrm{Pd}$, whereas the bcc alloy considered is $\mathrm{Nb}-\mathrm{Ta}$. None of the compositions modeled for any of these alloys show presence of ordered phases in their respective phase diagrams.

\section{Method validation}

To validate the accuracy of our DFT calculations and the methodology used to compute the thermodynamic quantities, we compare our results with that of some alloys already available in literature. In particular, we compare the order-disorder transition temperature, i.e., the temperature at which a binary 


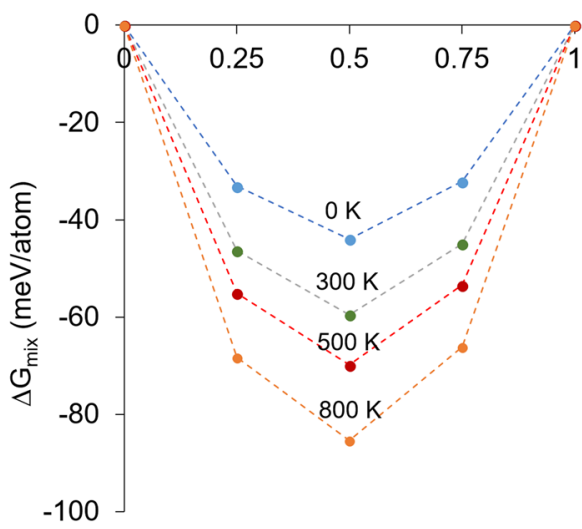

Au conc.

(a) Ag-Au

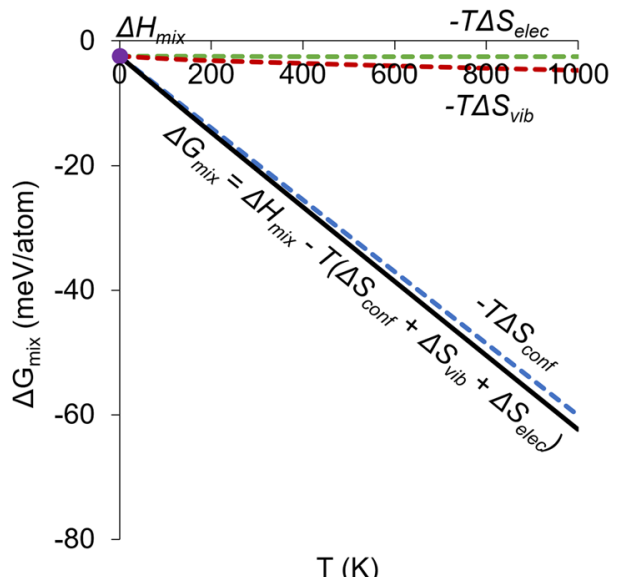

(c) $\mathrm{Nb}_{0.5}-\mathrm{Ta}_{0.5}$

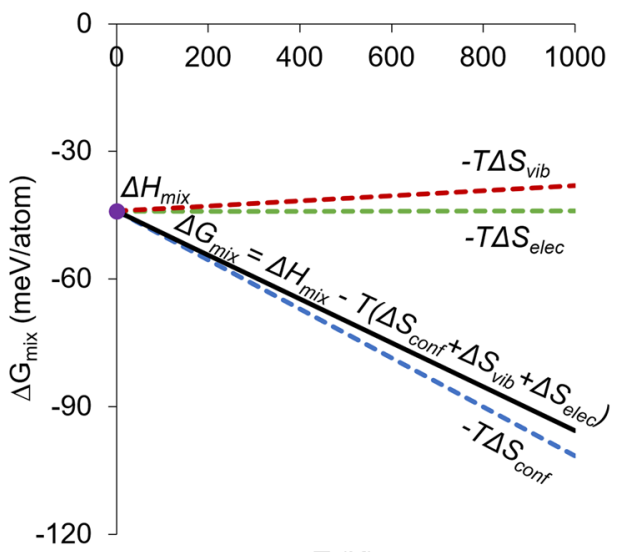

$\mathrm{T}(\mathrm{K})$

(b) $\mathrm{Ag}_{0.5}-\mathrm{Au}_{0.5}$

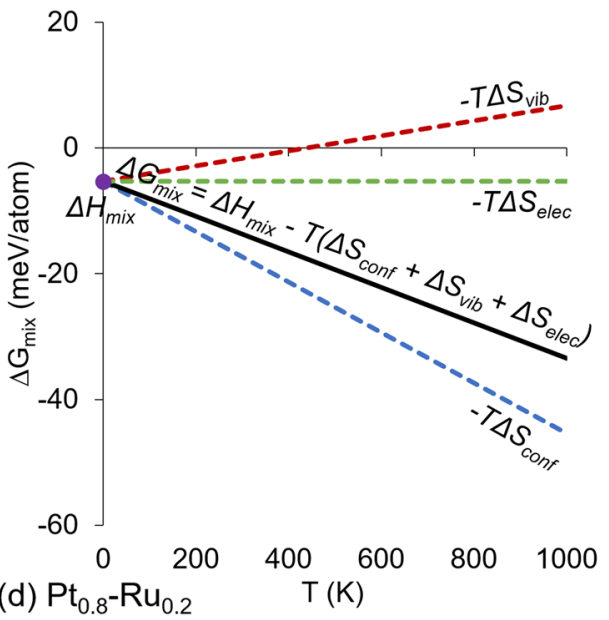

Fig. 2 a Convex hull of Ag-Au binary system showing solid-solution phase stability across the composition range. $\Delta G_{\text {mix }}$ vs $T$ plots for $\mathbf{b}$ $A g_{0.5}-\mathrm{Au}_{0.5}, \mathbf{c} \mathrm{Nb}_{0.5}-\mathrm{Ta}_{0.5}$, and $\mathbf{d} \mathrm{Pt}_{0.8}-\mathrm{Ru}_{0.2}$ in which $-T \Delta \mathrm{S}_{\mathrm{vib}},-T \Delta \mathrm{S}_{\text {conf }}$ and $-T \Delta \mathrm{S}_{\text {elec }}$ are shown to highlight the contribution of vibrational, configurational, and electronic entropies, respectively. While $\Delta S_{\text {vib }}$ contribution is negligible in $\mathbf{c}$, it is unfavorable in $\mathbf{b}$ and $\mathbf{d}$

alloy transforms from $\mathrm{L}_{0}$ ordered to disordered phase. Table 2 shows the comparison with the experimental and previous DFT predictions in $\mathrm{Cu}_{0.5}-\mathrm{Au}_{0.5}, \mathrm{Ni}_{0.5}-\mathrm{Fe}_{0.5}$ and $\mathrm{Ni}_{0.5}-\mathrm{Pt}_{0.5}$ systems. The temperature is computed by comparing the $\Delta G_{\text {mix }}$ (i.e., $\Delta H_{\text {mix }}-$ $\mathrm{T} \Delta S_{\text {conf }}-\mathrm{T} \Delta S_{\text {vib }}-\mathrm{T} \Delta S_{\text {elec }}$ ) between the ordered and the disordered structures for each of the alloys. The temperatures predicted for $\mathrm{Cu}-\mathrm{Au}, \mathrm{Ni}-\mathrm{Fe}$ and $\mathrm{Ni}-\mathrm{Pt}$ are $700 \mathrm{~K}, 700 \mathrm{~K}$, and $730 \mathrm{~K}$, which are in fairly good agreement with the experimental values of $683 \mathrm{~K}^{26}$ $618 \mathrm{~K}_{1}^{27}$ and $900 \mathrm{~K}_{1}^{28}$ respectively. Our calculated temperatures also compare well with the previous DFT results of $560 \mathrm{~K}^{4}$ and $755 \mathrm{~K}^{29}$ in $\mathrm{Cu}-\mathrm{Au}$ and $\mathrm{Ni}-\mathrm{Pt}$, respectively as shown in Table 2. We also compare $\Delta G_{\text {mix }}$ over a range of temperatures. Figure 1 shows the comparison of relative Gibbs free energy $\left(\Delta G_{\text {mix }}\right)$ and relative entropy $\left(\Delta S_{\text {mix }}\right)$ of $\mathrm{Ni}_{0.5}-\mathrm{Pt}_{0.5}$ between ordered and disordered structures with the DFT results from Shang et al. ${ }^{29}$ in Ni-Pt system from $0-1000 \mathrm{~K}$. The overall behaviors of both $\Delta G_{\text {mix }}$ (with and without configurational entropy) are in very close agreement. The close agreement with the previous works provides a validation to our calculations.

In what follows, each of the four cases described in Table 1 are discussed in detail. Relevant examples of binary solid solutions for the four cases are provided, and the contribution of the entropies towards the phase stability, or the formation of a miscibility gap, is illustrated. We show that, despite $\Delta H_{\text {mix }}<0$ for a given alloy, where the composition is expected to be stable in the form of a single phase solid solution, the phase diagram can surprisingly show a miscibility gap. Similarly, despite $\Delta H_{\text {mix }}>0$, where the composition is expected to be unstable, a stable single-phase solid solution can be observed. We show that both these unexpected observations highlight significant contributions of both types of entropies, and the phase stability of alloys may not be predicted simply based on $\Delta H_{\text {mix }}$ at least in some cases. While these two situations correspond to cases 3 and 4 in Table 1, we first present the results of cases 1 and 2 which behave in the manner expected on the basis of enthalpic arguments.

\section{Case 1 - Single-phase solid solution when $\Delta H_{\text {mix }} \leq 0$}

The $\mathrm{Ag}-\mathrm{Au}$ system has an isomorphous phase diagram, which shows complete solid solubility across the whole phase diagram. ${ }^{30}$ As a result, $\Delta H_{\text {mix }}$ is expected to be negative across the whole composition range. Figure $2 \mathrm{a}$ shows the convex hull for the $\mathrm{Ag}-\mathrm{Au}$ systems plotted for three alloy compositions: $0.25,0.5$, and 0.75 molar fraction of Au. The calculations indeed show that $\Delta H_{\text {mix }}<0$ for all three compositions illustrating stable $\mathrm{Ag}-\mathrm{Au}$ solid solutions. The effect of temperature is added by including the entropy contributions as shown in Fig. $2 a$.

Figure $2 \mathrm{~b}$ shows the $\Delta G_{\text {mix }}$ vs $T$ plot for $A g_{0.5}-A_{0.5}$ system. $\Delta G_{\text {mix }}$ shows the total free energy of the system over a given temperature range. To separate the individual contributions of the electronic, vibrational, and configurational entropies, $-T \Delta S_{\text {elec }}$ $-T \Delta S_{\text {vibr }}$ and $-T \Delta S_{\text {conf }}$ are also shown. It is found that the electronic entropy makes no significant contributions to the total free energy, whereas vibrational entropy makes a very minor 

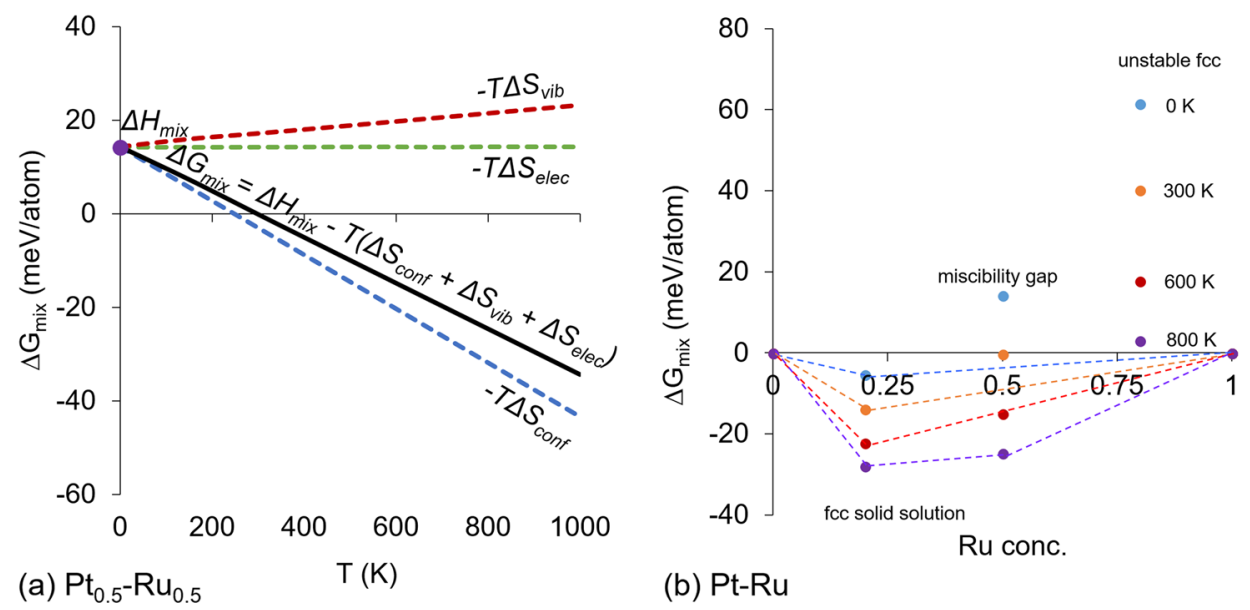

(b) Pt-Ru
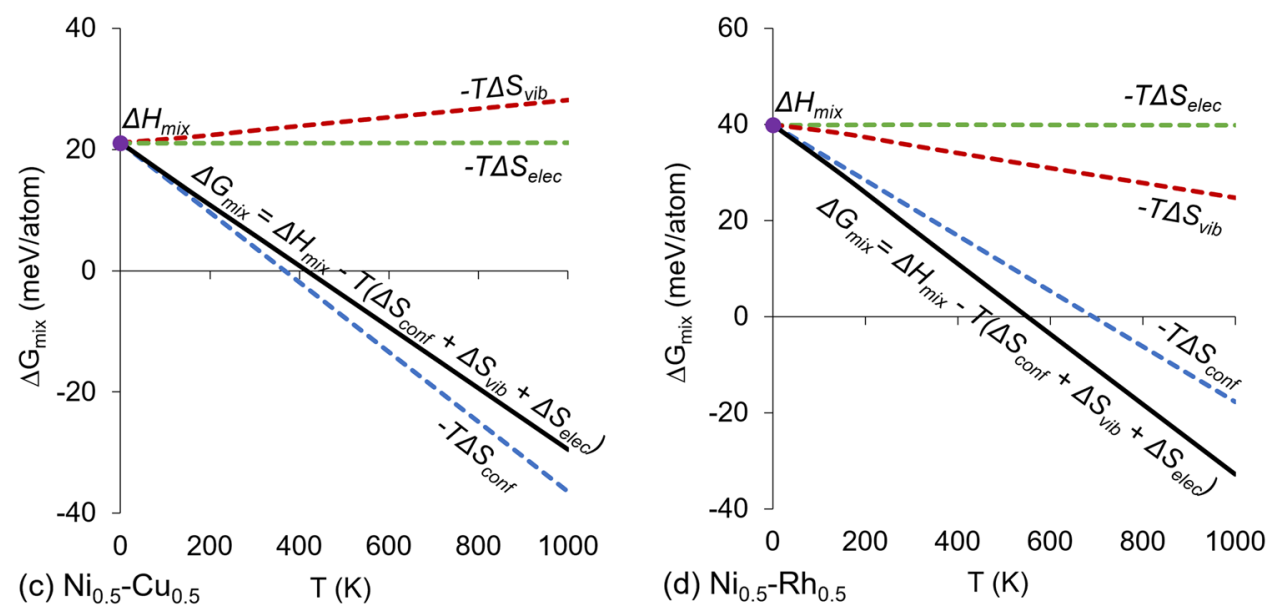


contributions of vibrational, configurational, and electronic entropies. b Convex hull of Pt-Ru binary system

contribution. Thus, the main entropy contribution to $\Delta G_{\text {mix }}$ is essentially configurational.

The $\mathrm{Nb}-\mathrm{Ta}$ solid solution also has an isomorphous phase diagram with complete solid solubility across the entire composition range. ${ }^{31}$ Fig. $2 c$ shows the $\Delta G_{\text {mix }}$ vs $T$ plot for the $\mathrm{Nb}_{0.5}-\mathrm{Ta}_{0.5}$ composition. The $\Delta G_{\text {mix }}$ plot shows a stable solid solution over the given temperature range. Similar to the $\mathrm{Ag}-\mathrm{Au}$ system, we find that configurational entropy contributes to the phase stability, whereas the electronic and vibrational entropy make no significant contributions. From these two binary systems, it is interesting to note that the phase stability of a system could be driven only by $\Delta H_{\text {mix }}$ and $\Delta S_{\text {conf, }}$ while $\Delta S_{\text {elec }}$ and $\Delta S_{\text {vib }}$ make no important contributions.

Figure $2 \mathrm{~d}$ shows the $\Delta G_{\text {mix }}$ vs $\mathrm{T}$ plot for $\mathrm{Pt}_{0.8}-\mathrm{Ru}_{0.2}$ system. The phase diagram of $\mathrm{Pt}-\mathrm{Ru}$ shows fcc single phase solid solution at $0.2 \mathrm{Ru}$ composition. ${ }^{32,33}$ As a result, similar to the above two systems, $\mathrm{Pt}_{0.8}-\mathrm{Ru}_{0.2}$ also shows negative $\Delta G_{\text {mix }}$, indicating a stable phase. The electronic entropy contribution is negligible as observed in the above two systems. However, in contrast to the above two systems, the influence of vibrational entropy is comparatively significant. While the $\Delta S_{\text {conf }}$ contribution is the same as in the two other systems, unlike these two systems, the $\mathrm{Pt}_{0.8}-\mathrm{Ru}_{0.2}$ system has a substantial positive $\Delta \mathrm{S}_{\mathrm{vib}}$ slope, as shown in Fig. $2 \mathrm{~d}$. In this case, the vibrational entropy disfavors the phase stability of the alloy. The contribution of $\Delta S_{\text {vib }}$ is $-43 \%$ to the total entropy, which is not negligible. From these three systems, we find that the vibrational entropy contributions could be significantly different among various alloys.
Case 2-Miscibility gap when $\Delta H_{\text {mix }} \geq 0$

The solid solutions that have $\Delta H_{\text {mix }} \geq 0$ are intuitively expected to be unstable. This instability can be revealed in the form of a miscibility gap in some phase diagrams. Only when the temperature is raised high enough does the miscibility gap close and the mixture transforms into a stable single-phase random solid solution. Here, such specific solid solutions are targeted to elucidate the significance of the two entropy contributions towards the formation of a miscibility gap.

Figure 3a shows the $\Delta G_{\text {mix }}$ vs $T$ plot for $\mathrm{Pt}_{0.5}-\mathrm{Ru}_{0.5}$ system where $\Delta G_{\text {mix, }}-T \Delta S_{\text {elec, }}-T \Delta S_{\text {vibr }}$ and $-T \Delta S_{\text {conf }}$ are shown. From DFT calculations, $\Delta H_{\text {mix }}$ is found to be $+14.2 \mathrm{meV} /$ atom. Since at $T=$ $0 \mathrm{~K}$, the $\Delta H_{\text {mix }}$ is positive, the solid solution is unstable. When the entropy contributions are added, the $\Delta \mathrm{G}_{\text {mix }}$ starts to decrease with temperature. The negative slope of $\Delta G_{\text {mix }}$ indicates that the solid solution will eventually become stable (i.e., $\Delta G_{\text {mix }}<0$ ), at higher temperatures. The contribution of electronic entropy is negligible for this system. However, the importance of the contribution of the two entropies $\left(\Delta S_{\text {vib }}\right.$ and $\left.\Delta S_{\text {conf }}\right)$ towards the stability of the solid solution is highlighted in this alloy. Figure 3a shows that $\Delta S_{\text {vib }}$ contributes unfavorably to the overall $\Delta G_{\text {mix }}$ as illustrated by the positive slope of the $-T \Delta S_{\text {vib }}$ plot.

Figure $3 \mathrm{~b}$ shows the convex-hull diagram for the Pt-Ru system. The relative stabilities of three compositions, i.e., at $0.2,0.5$ and 0.8 molar fraction $\mathrm{Ru}$, predicted by DFT calculations are shown. These predictions are in agreement with the phase diagram. The Pt-Ru phase diagram ${ }^{32,33}$ shows fcc single-phase solid solution in the range up to $0.38 \mathrm{Ru}$ and a miscibility gap beyond that composition 

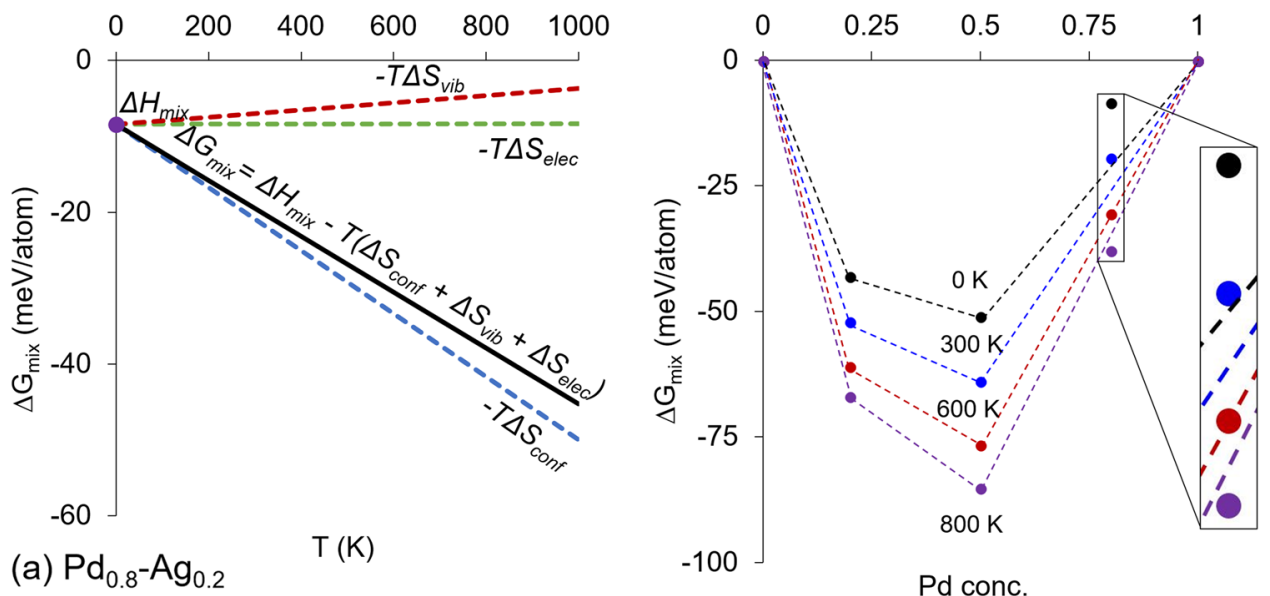

(b) Pd-Ag

Pd conc.

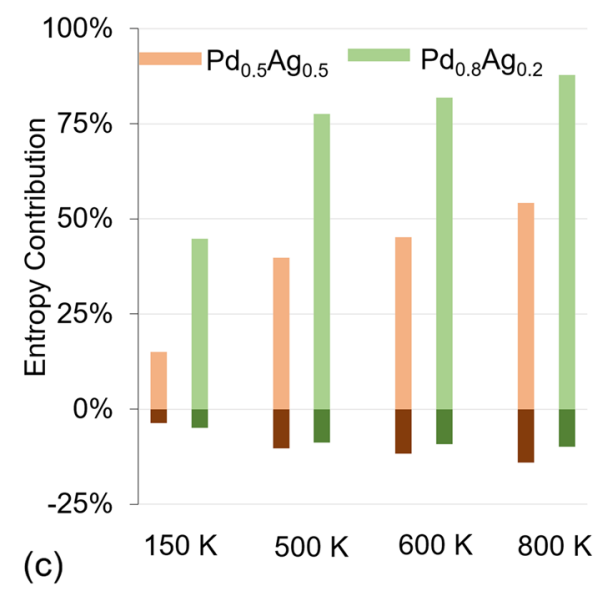

Fig. 4 a $\Delta G_{\text {mix }}$ vs $T$ for $\mathrm{Pd}_{0.8}-\mathrm{Ag}_{0.2}$ showing $-T \Delta S_{\mathrm{vib}}-T \Delta S_{\text {conf }}$ and $-T \Delta S_{\text {elec }}$ contributions. b Convex-hull diagram of Pd-Ag binary system. c Percentage entropy contribution in $\Delta G_{\text {mix }}$ for $\mathrm{Pd}_{0.5}-\mathrm{Ag}_{0.5}$ and $\mathrm{Pd}_{0.8}-\mathrm{Ag}_{0.2}$ at various temperatures. The entropy contribution is significantly higher in $\mathrm{Pd}_{0.8}-\mathrm{Ag}_{0.2}$ than $\mathrm{Pd}_{0.5}-\mathrm{Ag}_{0.5}$. The vibrational entropy contribution is minor in both as shown by the darker sub-bars

at room temperature. Figure $3 \mathrm{~b}$ shows a similar stability trend where the fcc solid solution is found to be stable at $0.2 \mathrm{Ru}$. For the $0.5 \mathrm{Ru}$ composition, the fcc is expected to be unstable at room temperature, and the predicted transition temperature to stable $\mathrm{fcc}$ is found to be $\sim 600 \mathrm{~K}$ which is in very good agreement with the phase diagram $(\sim 600 \mathrm{~K}))^{32,33}$ At $0.8 \mathrm{Ru}$ the fcc phase is found to be unstable, as shown by the positive $\Delta G_{\text {mix }}$ data points all of which lie above the tie line for the corresponding temperatures in Fig. $3 b$; this fcc phase instability is again consistent with the phase diagram.

To further emphasize the importance of the contributions of the two entropies, the $\Delta G_{\text {mix }}$ vs $T$ plots are presented for $\mathrm{Ni}_{0.5}-\mathrm{Cu}_{0.5}$ and $\mathrm{Ni}_{0.5}-\mathrm{Rh}_{0.5}$ systems in Fig. $3 c$, d, respectively. The $-T \Delta S_{\text {elec }}$ lines are flat for both systems showing the negligible contribution of the electronic entropy. The predicted transition temperature of $\mathrm{Ni}_{0.5}-\mathrm{Cu}_{0.5}$ is $400 \mathrm{~K}$ shown in Fig. $3 \mathrm{C}$ which is in qualitative agreement with the phase diagram (i.e., $\sim 620 \mathrm{~K}$ ). ${ }^{34} \mathrm{The} \mathrm{Ni}_{0.5}-\mathrm{Cu}_{0.5}$ has similar positive $\Delta S_{\text {vib }}$ slope compared to $\mathrm{Pt}_{0.5}-\mathrm{Ru}_{0.5}$ indicating that the contribution of the vibrational entropy in $\mathrm{Ni}_{0.5}-\mathrm{Cu}_{0.5}$ is same as that in $\mathrm{Pt}_{0.5}-\mathrm{Ru}_{0.5}$. Consequently, the slope of the total Gibbs free energy of mixing is also same between the two alloys. In contrast, we find that the $\Delta \mathrm{S}_{\mathrm{vib}}$ in $\mathrm{Ni}_{0.5}-\mathrm{Rh}_{0.5}$ has negative slope; this means, the contribution of $\Delta S_{\text {vib }}$ is favorable to its phase stability as shown in Fig. $3 \mathrm{~d}$. The predicted miscibility gap transition temperature for $\mathrm{Ni}_{0.5}-\mathrm{Rh}_{0.5}$ is $575 \mathrm{~K}$ as shown in Fig. $3 \mathrm{~d}$; here, $\Delta S_{\text {vib }}$ actually favors the formation of a single phase as shown by its negative slope, thereby adding to the contribution of
$\Delta S_{\text {conf }}$ towards the overall $\Delta G_{\text {mix }}$. The $\Delta S_{\text {vib }}$ contribution in $\mathrm{Ni}_{0.5}-\mathrm{Rh}_{0.5}$ is $+21 \%$ whereas in other two systems $\left(\mathrm{Pt}_{0.5}-\mathrm{Ru}_{0.5}\right.$ and $\left.\mathrm{Ni}_{0.5}-\mathrm{Cu}_{0.5}\right)$ it is $-18 \%$ and $-14 \%$, as shown in Fig. $6 \mathrm{~b}$. With respect to the phase diagram, the transition temperature is $\sim 350 \mathrm{~K}$ for $\mathrm{Ni}_{0.5}-\mathrm{Rh}_{0.5},{ }_{3}$ which is again in qualitative agreement with our calculations. Thus, in accord with the observation in case 1, we find that the entropy contributions could be different among various systems. In addition, we find that the vibrational entropy could favor or disfavor phase stability of alloys.

Case 3-Miscibility gap when $\Delta H_{\text {mix }} \leq 0$

The behavior of the above two cases is generally intuitive, i.e., when $\Delta H_{\text {mix }}$ is $<0$, a stable phase is expected, whereas when $\Delta H_{\text {mix }}>0$, an unstable phase or a miscibility gap is expected. However, cases 3 and 4 can seem unintuitive, i.e., even when the $\Delta H_{\text {mix }}<0$, the phase diagram can show a miscibility gap. Similarly, when $\Delta H_{\text {mix }}>0$, the system may be stable, and a miscibility gap can be absent. In both these cases, we show that entropy plays a crucial role in dictating the phase stability, and the contributions of each of the three entropies are disentangled.

The $\mathrm{Pd}-\mathrm{Ag}$ phase diagram shows a miscibility gap in the range 0.65-0.98 molar fraction $\mathrm{Pd}^{36,37}$ The miscibility gap transition temperature peaks at $\sim 0.85 \mathrm{Pd}$ is $620 \mathrm{~K}$. Consequently, $\mathrm{Pd}_{0.8}-\mathrm{Ag}_{0.2}$ is expected to be unstable with respect to the two pure elements. However, we find that DFT predicts $\Delta H_{\text {mix }}<0$, indicating a stable solid solution. This 'pseudo' stability is shown in Fig. $4 a$, where $\Delta G_{\text {mix }}$ decreases with the increase in temperature. The 

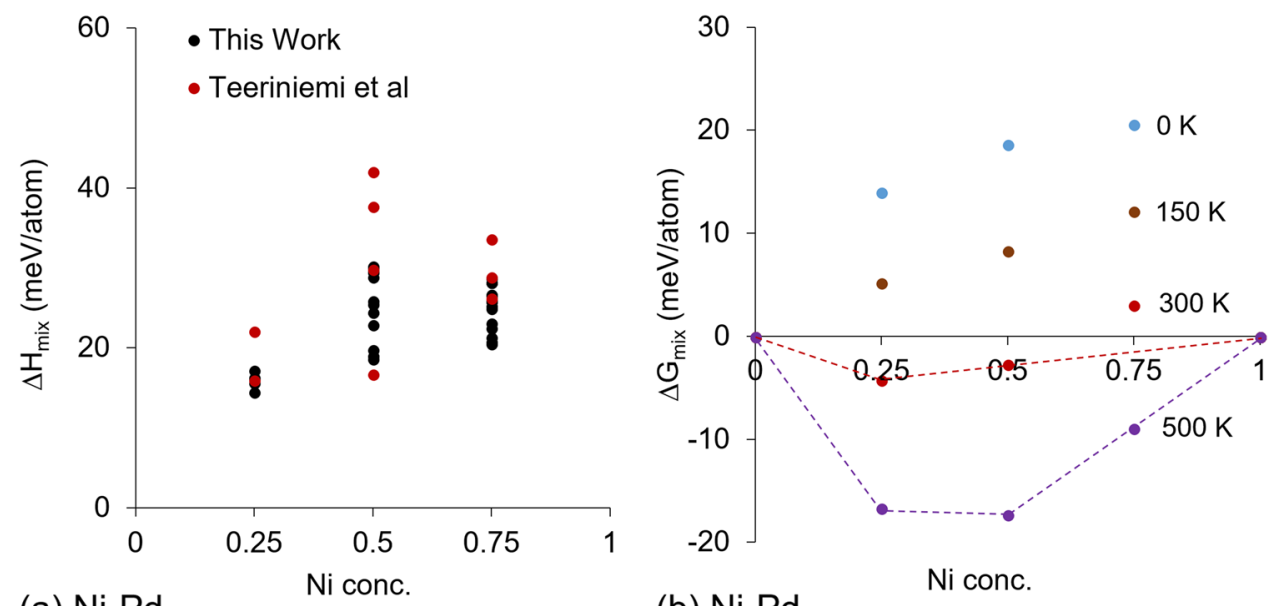

(a) $\mathrm{Ni}-\mathrm{Pd}$

(b) $\mathrm{Ni}-\mathrm{Pd}$

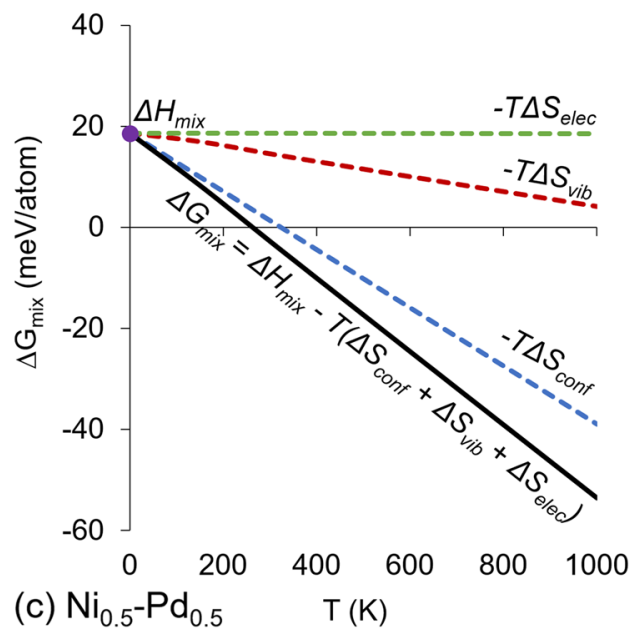

Fig. 5 a Comparison of $\Delta H_{\text {mix }}$ with Teeriniemi et al. ${ }^{38}$ showing $\Delta H_{\text {mix }}>0$ from both studies. b Convex-hull diagram of Ni-Pd binary system. c $\Delta G_{\text {mix }}$ vs $T$ plots for $\mathrm{Ni}_{0.5}-\mathrm{Pd}_{0.5}$ system showing contributions of vibrational, configurational and electronic entropies

contribution of $\Delta S_{\text {elec }}$ is negligible and $\Delta S_{\text {vib }}$ slightly disfavors phase stability shown by the positive slope in Fig. 4 a. The $\Delta S_{\text {conf }}$ provides the pseudo stability that leads to the decrease in $\Delta G_{\text {mix }}$ with temperature. Here, the $\Delta G_{\text {mix }}$ vs $T$ plot is rather misleading as it does not show the evidence of the miscibility gap.

The correct phase behavior is revealed in the convex-hull diagram. Figure $4 \mathrm{~b}$ shows the convex-hull for $\mathrm{Pd}-\mathrm{Ag}$ system, where $\Delta G_{\text {mix }}$ for three compositions, i.e., $0.2,0.5$, and 0.8 molar fraction Pd, are plotted at $0 \mathrm{~K}, 300 \mathrm{~K}, 600 \mathrm{~K}$, and $800 \mathrm{~K}$. We find that for all four temperatures, the free energies of 0.2 and 0.5 solid solution compositions lie on the convex-hull, indicating their phase stability, which agrees with the phase diagram. In contrast, the free energies of $0.8 \mathrm{Pd}$ composition for $0 \mathrm{~K}$ and $300 \mathrm{~K}$ clearly lie above their tie lines, thereby indicating its instability. The free energy decreases with increasing temperature. By $\sim 600 \mathrm{~K}$, it lies almost on the tie line, indicating that the system is just starting to stabilize. By $800 \mathrm{~K}$, the free energy lies well below the tie line, showing its complete stability at higher temperatures. The transition temperature from phase separation to the stable random solid solution is just above $600 \mathrm{~K}$, which is in very good agreement with the phase diagram which also shows the transition temperature to be $\sim 600 \mathrm{~K}^{37}$ While the increase in phase stability with temperature is not a new observation, this example reinforces the point that simply relying on $\Delta H_{\text {mix }}$ may not be sufficient in shallow $\Delta H_{\text {mix }}$ solid solutions and including entropy contributions could be crucial.
Since the phase stability of $\mathrm{Pd}_{0.8}-\mathrm{Ag}_{0.2}$ is achieved by raising the temperature, the decrease in the free energy can only be due to the entropy contributions. Figure $4 c$ shows the percentage contributions of the vibrational and configurational entropies to the total free energy for $\mathrm{Pd}_{0.5}-\mathrm{Ag}_{0.5}$ and $\mathrm{Pd}_{0.8}-\mathrm{Ag}_{0.2}$ compositions at $150 \mathrm{~K}, 500 \mathrm{~K}, 600 \mathrm{~K}$, and $800 \mathrm{~K}$. We find that the total entropy contribution is significantly higher for $\mathrm{Pd}_{0.8}-\mathrm{Ag}_{0.2}$ than $\mathrm{Pd}_{0.5}-\mathrm{Ag}_{0.5}$, whereas in $\mathrm{Pd}_{0.8}-\mathrm{Ag}_{0.2}$ it is over $75 \%$, that in $\mathrm{Pd}_{0.5}-\mathrm{Ag}_{0.5}$ is only $\sim 40 \%$. This enhanced entropy contribution in $\mathrm{Pd}_{0.8}-\mathrm{Ag}_{0.2}$ is the reason that drives the stability of $\mathrm{Pd}_{0.8}-\mathrm{Ag}_{0.2}$ at higher temperatures. However, by separating the contributions of the two entropies, we find that the vibrational contribution (shown by darker bars in Fig. 4c) is very small in both alloys for all four temperatures, and the primary contribution is configurational.

Case 4-Single-phase solid solution when $\Delta H_{\text {mix }} \geq 0$

The final case from Table 1 is the formation of a single-phase solid solution even when $\Delta H_{\text {mix }}$ is predicted to be positive. While $\Delta H_{\text {mix }}$ is greater than 0 for both cases 2 and 4 , there is presence of a miscibility gap in case 2 in contrast to formation of a solid solution in case 4 . Thus, in case 4 , simply relying on $\Delta H_{\text {mix }}$ would predict phase instability (or the presence of a miscibility gap). By taking example of $\mathrm{Ni}-\mathrm{Pd}$ system, we show that incorporating the entropy contribution provides the correct phase stability in agreement with the $\mathrm{Ni}-\mathrm{Pd}$ phase diagram. 
Table 3. Electronic entropy of metals and alloys at $1000 \mathrm{~K}$

\begin{tabular}{|c|c|c|c|c|}
\hline Metal 1 & Metal 2 & Alloy & $\Delta S_{\text {elec }}(\mathrm{meV} / \mathrm{K} /$ atom $)$ & $T \Delta S_{\text {elec }}(\mathrm{meV} /$ atom $)$ \\
\hline $4.4 \times 10^{-5}(\mathrm{Ag})$ & $5.0 \times 10^{-5}(\mathrm{Au})$ & $4.6 \times 10^{-5}\left(\mathrm{Ag}_{0.5}-\mathrm{Au}_{0.5}\right)$ & $-7.0 \times 10^{-7}\left(\mathrm{Ag}_{0.5}-\mathrm{Au}_{0.5}\right)$ & $-7.0 \times 10^{-4}\left(\mathrm{Ag}_{0.5}-\mathrm{Au}_{0.5}\right)$ \\
\hline $2.2 \times 10^{-4}(\mathrm{Nb})$ & $2.1 \times 10^{-4}(\mathrm{Ta})$ & $2.2 \times 10^{-4}\left(\mathrm{Nb}_{0.5}-\mathrm{Ta}_{0.5}\right)$ & $4.8 \times 10^{-6}\left(\mathrm{Nb}_{0.5}-\mathrm{Ta}_{0.5}\right)$ & $4.8 \times 10^{-3}\left(\mathrm{Nb}_{0.5}-\mathrm{Ta}_{0.5}\right)$ \\
\hline $2.3 \times 10^{-4}(\mathrm{Pt})$ & $1.5 \times 10^{-4}(\mathrm{Ru})$ & $2.1 \times 10^{-4}\left(\mathrm{Pt}_{0.8}-\mathrm{Ru}_{0.2}\right)$ & $-8.6 \times 10^{-6}\left(\mathrm{Pt}_{0.8}-\mathrm{Ru}_{0.2}\right)$ & $-8.6 \times 10^{-3}\left(\mathrm{Pt}_{0.8}-\mathrm{Ru}_{0.2}\right)$ \\
\hline $2.3 \times 10^{-4}(\mathrm{Pt})$ & $1.5 \times 10^{-4}(\mathrm{Ru})$ & $1.9 \times 10^{-4}\left(\mathrm{Pt}_{0.5}-\mathrm{Ru}_{0.5}\right)$ & $-3.6 \times 10^{-6}\left(\mathrm{Pt}_{0.5}-\mathrm{Ru}_{0.5}\right)$ & $-3.6 \times 10^{-3}\left(\mathrm{Pt}_{0.5}-\mathrm{Ru}_{0.5}\right)$ \\
\hline $2.6 \times 10^{-4}(\mathrm{Pd})$ & $4.4 \times 10^{-5}(\mathrm{Ag})$ & $2.0 \times 10^{-4}\left(\mathrm{Pd}_{0.8}-\mathrm{Ag}_{0.2}\right)$ & $-1.2 \times 10^{--5}\left(\mathrm{Pd}_{0.8}-\mathrm{Ag}_{0.2}\right)$ & $-1.2 \times 10^{-2}\left(\mathrm{Pd}_{0.8}-\mathrm{Ag}_{0.2}\right.$ \\
\hline $3.4 \times 10^{-4}(\mathrm{Ni})$ & $2.6 \times 10^{-4}(\mathrm{Pd})$ & $3.1 \times 10^{-4}\left(\mathrm{Ni}_{0.5}-\mathrm{Pd}_{0.5}\right)$ & $1.4 \times 10^{-5}\left(\mathrm{Ni}_{0.5}-\mathrm{Pd}_{0.5}\right)$ & $1.4 \times 10^{-2}\left(\mathrm{Ni}_{0.5}-\mathrm{Pd}_{0.5}\right)$ \\
\hline
\end{tabular}

Table 4. Vibrational entropy of metals and alloys at $1000 \mathrm{~K}$

\begin{tabular}{|c|c|c|c|c|}
\hline \multicolumn{3}{|c|}{$S_{\text {vib }}(\mathrm{meV} / \mathrm{K} /$ atom $)$} & $\Delta S_{\text {vib }}(\mathrm{meV} / \mathrm{K} /$ atom $)$ & $T \Delta S_{\text {vib }}$ (meV/atom) \\
\hline $1.01(\mathrm{Ag})$ & $1.10(\mathrm{Au})$ & $1.05\left(\mathrm{Ag}_{0.5}-\mathrm{Au}_{0.5}\right)$ & $-5.9 \times 10^{-3}\left(\mathrm{Ag}_{0.5}-\mathrm{Au}_{0.5}\right)$ & $-5.95\left(\mathrm{Ag}_{0.5}-\mathrm{Au}_{0.5}\right)$ \\
\hline $0.99(\mathrm{Pt})$ & $0.79(\mathrm{Ru})$ & $0.94\left(\mathrm{Pt}_{0.8}-\mathrm{Ru}_{0.2}\right)$ & $-1.2 \times 10^{-2}\left(\mathrm{Pt}_{0.8}-\mathrm{Ru}_{0.2}\right)$ & $-12.00\left(\mathrm{Pt}_{0.8}-\mathrm{Ru}_{0.2}\right)$ \\
\hline $0.99(\mathrm{Pt})$ & $0.79(\mathrm{Ru})$ & $0.88\left(\mathrm{Pt}_{0.5}-\mathrm{Ru}_{0.5}\right)$ & $-8.9 \times 10^{-3}\left(\mathrm{Pt}_{0.5}-\mathrm{Ru}_{0.5}\right)$ & $-8.92\left(\mathrm{Pt}_{0.5}-\mathrm{Ru}_{0.5}\right)$ \\
\hline $0.91(\mathrm{Pd})$ & $1.01(\mathrm{Ag})$ & $0.93\left(\mathrm{Pd}_{0.8}-\mathrm{Ag}_{0.2}\right)$ & $-4.7 \times 10^{-3}\left(\mathrm{Pd}_{0.8}-\mathrm{Ag}_{0.2}\right)$ & $-4.72\left(\mathrm{Pd}_{0.8}-\mathrm{Ag}_{0.2}\right)$ \\
\hline $0.78(\mathrm{Ni})$ & $0.91(\mathrm{Pd})$ & $0.86\left(\mathrm{Ni}_{0.5}-\mathrm{Pd}_{0.5}\right)$ & $1.5 \times 10^{-2}\left(\mathrm{Ni}_{0.5}-\mathrm{Pd}_{0.5}\right)$ & $14.58\left(\mathrm{Ni}_{0.5}-\mathrm{Pd}_{0.5}\right)$ \\
\hline
\end{tabular}

In the calculation of $\Delta H_{\text {mix }}$ for random alloys, the total energy of a given alloy composition could significantly vary due to the multiple ways of randomly distributing atoms during the creation of initial crystal structures. Figure 5 a shows $\Delta H_{\text {mix }}$ of multiple structures for three compositions: $\mathrm{Ni}_{0.25} \mathrm{Pd}_{0.75}, \mathrm{Ni}_{0.5} \mathrm{Pd}_{0.5}$ and $\mathrm{Ni}_{0.75} \mathrm{Pd}_{0.25}$. Our calculations predict $\Delta H_{\text {mix }}>0$ for all structures. These results are in agreement with previous DFT calculations from Teeriniemi et al. ${ }^{38}$ as shown in Fig. 5a. However, both results are in contrast to the $\mathrm{Ni}-\mathrm{Pd}$ phase diagram that shows a complete solid solubility across the entire composition range. ${ }^{39}$

The correct structure, a stable random solid solution, is predicted when entropy contributions are included. Figure $5 b$ shows the convex-hull diagram formed using the three compositions. The $\Delta H_{\text {mix }}$ structures used in Fig. 5b are the lowest energy structures from Fig. 5a for the corresponding compositions. As expected, the stability increases with the increase in temperature. $\mathrm{Ni}_{0.25}-\mathrm{Pd}_{0.75}$ and $\mathrm{Ni}_{0.5}-\mathrm{Pd}_{0.5}$ are stable at $300 \mathrm{~K}$, whereas $\mathrm{Ni}_{0.75}-\mathrm{Pd}_{0.25}$ becomes stable above $300 \mathrm{~K}$. By $500 \mathrm{~K}$, all three compositions are stable, as shown.

Figure $5 \mathrm{c}$ shows the $\Delta G_{\text {mix }}$ vs $T$ plot for $\mathrm{Ni}_{0.5}-\mathrm{Pd}_{0.5}$ where the individual contributions of $-T \Delta S_{\text {elec }}-T \Delta S_{\text {vib }}$ and $-T \Delta S_{\text {conf }}$ are shown. We find that electronic entropy contributions are negligible whereas vibrational entropy has a negative slope, and it contributes favorably towards the phase stability and the overall $\Delta G_{\text {mix }}$ along with $\Delta S_{\text {conf. It }}$ is important to point out that while $\Delta \mathrm{G}_{\text {mix }}$ becomes negative only above $\sim 240 \mathrm{~K}$, the positive value of $\Delta G_{\text {mix }}$ below $240 \mathrm{~K}$ does not indicate a miscibility gap, unlike Fig. 3 . The positive $\Delta G_{\text {mix }}$ originates from $\Delta H_{\text {mix }}$ which is greater than 0 to begin with. Only with the addition of entropy, the complete picture emerges, as revealed by the convex-hull diagram in Fig. 5b. Thus, Pd-Ag and Ni-Pd systems discussed under cases 3 and 4 collectively highlight the importance of entropy contributions towards predicting the correct phase stability of these alloys.
Finally, we summarize the individual contributions of electronic, vibrational and configurational entropies for all the systems discussed above. Tables 3 and 4 show the electronic and vibrational entropy respectively of metals and alloys at $1000 \mathrm{~K}$. Figure $6 \mathrm{a}-\mathrm{c}$ shows the percentages of electronic, vibrational and configurational entropy contribution to the total entropy at $1000 \mathrm{~K}$, where the negative (positive) sign indicates that electronic and vibrational entropy disfavor (favor) the phase stability or free energy. We find that the electronic entropy contributions are negligible in all alloys (of the order of $10^{-2} \mathrm{meV} / \mathrm{atom}$ ), and they are not comparable to vibrational and configurational entropy contributions as shown in Fig. 6. The vibrational entropy is comparable to that of the configurational entropy, but it shows large variation among the alloys. For example, in $\mathrm{Pt}_{0.8}-\mathrm{Ru}_{0.2}$ system, the vibrational entropy has an opposite contribution to the configurational entropy by over $40 \%$ as shown in Fig. $6 \mathrm{~b}$. In contrast, in $\mathrm{Ni}_{0.5}-\mathrm{Pd}_{0.5}$ and $\mathrm{Ni}_{0.5}-\mathrm{Rh}_{0.5}$ it favors the phase stability and has a favorable contribution of more than $20 \%$. While the unfavorable contribution would tend to destabilize the system, creating propensity to miscibility gap, the favorable contribution would help in phase stability.

\section{DISCUSSION}

These results show that in the narrow-range $\Delta H_{\text {mix }}$ solid solutions, the contribution of $\Delta S_{\text {mix }}$ is important, and cannot be ignored, unlike that in the ordered structures. For example, while $\Delta H_{\text {mix }}$ in $\mathrm{Ni}_{0.5}-\mathrm{Rh}_{0.5}$ at $575 \mathrm{~K}$ is $+40 \mathrm{meV} /$ atom, the total $\Delta S_{\text {mix }}$ is almost the same, i.e., $40 \mathrm{meV} / \mathrm{atom}$ (Fig. $3 \mathrm{~d}$ ), illustrating that the contribution of $\Delta S_{\text {mix }}$ is large and quantitatively similar to $\Delta H_{\text {mix }}$. The important contribution of $\Delta S_{\text {mix }}$ is further demonstrated in a quaternary alloy, $\mathrm{Fe}-\mathrm{Ni}-\mathrm{Co}-\mathrm{Cr}$. The calculated $\Delta H_{\text {mix }}$ of this alloy is $+65 \mathrm{meV} /$ atom. This is consistent with previous DFT calculations from Gao et al. ${ }^{40}$ and Troparevsky et al. ${ }^{20}$ Based on such large positive $\Delta H_{\text {mix }}$ this 

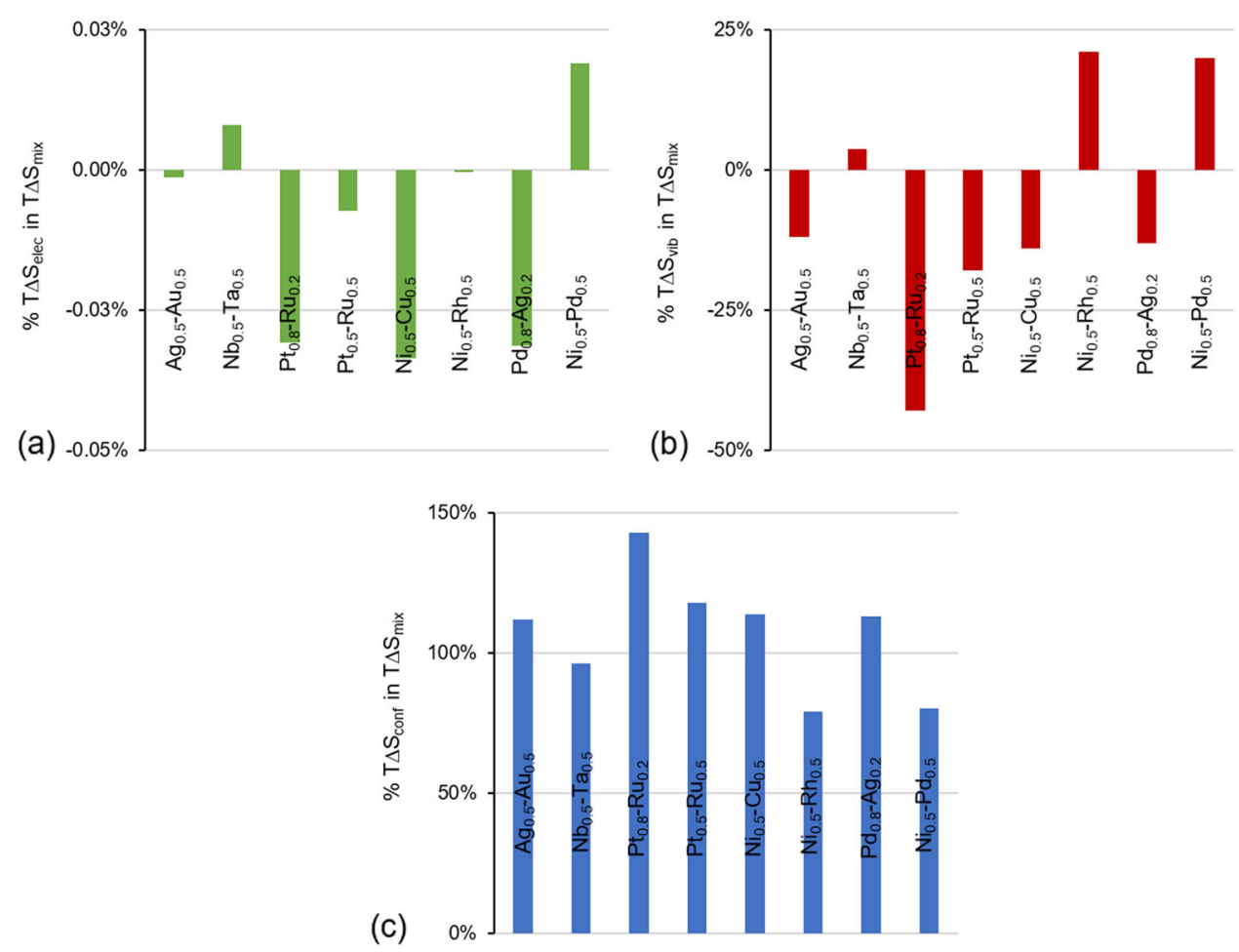

Fig. 6 a Percentages of electronic entropy $\left(T \Delta S_{\text {elec }}\right)$, b vibrational entropy $\left(T \Delta S_{\text {vib }}\right)$, and c configurational entropy contributions $\left(T \Delta S_{\text {conf }}\right)$ in total entropy $\left(T \Delta S_{\text {mix }}\right)$ of the binary solid solutions

alloy is expected to be unstable, however, experimentally it is found to be single phase stable. ${ }^{41}$ The positive $\Delta H_{\text {mix }}$ is flipped to negative (and stable) $\Delta G_{\text {mix }}$ via entropy contributions. Our calculations (in agreement with Gao et al.) show that $\Delta S_{\text {vib }}$ has a contribution of over $27 \%$ to the total entropy at room temperature (the rest being configurational entropy). Without the contribution of the vibrational entropy, the stability of this phase would occur at $\sim 600 \mathrm{~K}$, whereas experimentally it is stable at room temperature. These calculations thus illustrate the importance of including entropy contributions towards the correct prediction of phase behaviors.

Our calculations also show that the contribution of $\Delta S_{\text {vib }}$ can be significantly different among the systems. While in some systems $\Delta S_{\text {vib }}$ disfavors the phase stability (such as in $\mathrm{Pt}_{0.8}-\mathrm{Ru}_{0.2}, \mathrm{Pt}_{0.5}-\mathrm{Ru}_{0.5}$ and $\mathrm{Ni}_{0.5}-\mathrm{Cu}_{0.5}$ ) in others it favors the phase stability (e.g., $\mathrm{Ni}_{0.5}-\mathrm{Rh}_{0.5}$ and $\left.\mathrm{Ni}_{0.5}-\mathrm{Pd}_{0.5}\right) ; \Delta \mathrm{S}_{\text {vib }}$ can have very little contribution as well, as observed in $\mathrm{Nb}_{0.5}-\mathrm{Ta}_{0.5}$. It has been speculated that there could be multiple reasons behind the different behaviors, such as electronegativity difference between the elements, atomic radius difference, electron-to-atom ratio, or the difference in the atomic masses. ${ }^{1}$ The vibrational entropy is the measure of stiffness of the atomic bonds; the softer the bonds, the higher the vibrational entropy. ${ }^{5}$ The effect of these factors fundamentally lies in the phonon dispersion curves which provide the frequencies of optical phonon modes. An alloy with softer bonds would have lower frequencies and higher entropy. While significant progress has been made in understanding the contributions to vibrational entropy, the contributions of each of these factors to the phonon dispersion curves is still not fully understood; our future work will focus in this direction.

Our calculations also show that electronic entropy has minor contribution to the total entropy. Similar observations were made previously on three quaternary systems, i.e., fcc $\mathrm{Co}-\mathrm{Cr}-\mathrm{Fe}-\mathrm{Ni}$, bcc Mo-Nb-Ta-W and hcp Co-Os-Re-Ru. ${ }^{40}$ Via DFT calculations, it was shown that the total entropy of these systems was of the order of $10^{-2} \mathrm{meV} /$ atom or less, and the $\Delta S_{\text {elec }}$ was negligible.
Thus, these calculations seem to indicate a greater importance of vibrational and configurational entropies compared to electronic. While three entropy contributions are considered in this work, the magnetic entropy could have an impact as well. ${ }^{42}$ Previous work has shown that the Curie temperature can be tuned by controlling the $\mathrm{Cr}$ concentration in $\mathrm{Fe}-\mathrm{Co}-\mathrm{Cr}-\mathrm{Ni}$ HEA. ${ }^{42}$ Understanding the individual contribution of various entropies is warranted.

In conclusion, this work shows that in the shallow-range $\Delta H_{\text {mix }}$ solid solutions, relying solely on enthalpy may not lead to correct prediction of phase stability, and including entropy contributions are critical. By disentangling the entropy contributions in various solid solutions, our calculations show that the two entropies (vibrational and configurational) can either destabilize or can collectively contribute to stabilize the solid solutions. Our calculations also show that electronic entropy contributions may be less significant compared to vibrational and configurational entropies towards the phase stability of binary random solid solutions. This work advances the understanding on thermodynamics of HEAs, and contributes towards building a theoretical framework for computational prediction of stable multi-elemental single-phase HEAs in future.

\section{METHODS}

First-principles calculations are performed with density functional theory (DFT) using Vienna Ab-initio Software Package (VASP). ${ }^{43}$ The Projector Augmented Wave (PAW) method is used with standard GGA-PBE exchange-correlation function. ${ }^{44}$ For structure relaxation, unit cells are sampled by Brillouin zones with a $4 \times 4 \times 4$ Monkhorst-Pack (MP) ${ }^{45}$ mesh with the $500 \mathrm{eV}$ energy cut-off of the wave function. The energy convergence criterion of the electronic self-consistency is chosen as $10^{-8} \mathrm{eV}$ for all the calculations.

Random structures are created using the special quasi-random structure (SQS) method. ${ }^{46} \mathrm{~A}$ SQS method calculates correlation functions of a finite cell and compares them to those of an infinite random system. The differences in the correlation functions can be used to quantify the randomness in the finite cell. The purpose of the SQS algorithm is to minimize the difference in the correlation functions. Thus, SQSs are 
believed to give a good approximation to near-randomness in solid solution alloys.

The eight-atom (SQS-8), 16-atom (SQS-16) and 32-atom (SQS-32) are used in the present study to predict the phase stability of various random binary systems. We have checked that the number of atoms in the SQS structures does not significantly affect the prediction of phase stability. The convergence is quantified by calculating the error bars as shown in Supplementary Information (see S1). The correlations in 32-atom (SQS-32) for $\mathrm{A}_{0.25}-\mathrm{B}_{0.75}$ and $\mathrm{A}_{0.5}-\mathrm{B}_{0.5}$ compositions are given in the Supplementary Information under S2. The widely used Alloy Theoretic Automated Toolkit (ATAT) is used to build the SQS structures. ${ }^{47}$

The supercell method is used for phonon calculations. ${ }^{5}$ The VASP code is used to calculate the real space force constants of supercells, and the PHONOPY ${ }^{48}$ code is used to calculate the phonon frequencies from the force constants on a supercell consisting of at least 32 atoms in all systems. In order to get the force-constant matrices for each binary system, every atom is displaced by a finite displacement of $0.01 \AA \AA$ in $x$-, $y$ - and $z$-direction. For 32 atoms, 192 sets of atomic positions with displacement employed to each random alloy. Strict energy convergence criteria of $\left(10^{-8} \mathrm{eV}\right)$ and $4 \times$ $4 \times 4$ k-points were used for the force constant calculations. After getting the force-constant matrices, the dynamical matrix is built for different qvectors in the Brillouin zone. The eigenvalues of phonon frequencies and eigenvectors of phonon modes are found by solving the dynamical matrix. The thermodynamic properties require summations over the phonon eigenvectors which is implemented in the PHONOPY code. The theory on the phonon calculations is discussed in Supplementary Information (see S3). We have checked the mechanical stability of all systems, and no imaginary modes are observed in the modeled structures. The phonon band structures figures for all the studied systems have also been added to the Supplementary Information under S4. The electronic entropy has been calculated using Fermi-Dirac distribution. The theory of electronic entropy is discussed in Supplementary Information (see S5).

In order to illustrate the phase stability, the change in Gibbs free energy of mixing is calculated for all binary systems. $\Delta G_{\text {mix }}$ is the difference of Gibbs free energy between an alloy and the individual elements. The expression for change in Gibbs free energy is, $\Delta G_{\text {mix }}=\Delta H_{\text {mix }}-T\left(\Delta S_{\text {conf }}+\right.$ $\Delta S_{\text {vib }}+\Delta S_{\text {elec }}$ ), where $\Delta H_{\text {mix }}$ is the enthalpy of mixing, $T$ is the absolute temperature, $\Delta S_{\text {conf, }} \Delta S_{\text {vib }}$ and $\Delta S_{\text {elec }}$ are the difference of configurational, vibrational and electronic entropy between alloy and individual elements. $\Delta S_{\text {conf }}$ is calculated using the standard equation, i.e., $\Delta S_{\text {conf }}=\Sigma x \ln x$, where $x$ is the number of elements in an alloy.

The relativistic effect of spin-orbit coupling (SOC) is important for heavier elements and its significance has been addressed by Xie et al. ${ }^{49}$ Their study showed that with SOC, the energies are found to be lowered by $20 \mathrm{meV} /$ atom in $\mathrm{U}-\mathrm{Zr}$ alloy. We also performed phase stability calculations using SOC. However, no significant difference in the transition temperature prediction was observed. A comparison of the transition temperatures between $\mathrm{SOC}$ and non-SOC calculations is provided in the Supplementary Information (see S6).

\section{DATA AVAILABILITY}

The authors declare that all data supporting the findings of this study are available within the paper and its supplementary information files.

\section{ACKNOWLEDGEMENTS}

This work was supported as part of the Energy Dissipation to Defect Evolution (EDDE), an Energy Frontier Research Center funded by the US Department of Energy, Office of Science, Basic Energy Sciences. We acknowledge the support of computational resources from Advanced Research Computing Center (ARCC) at the University of Wyoming. We also thank Richard Hennig for fruitful discussions on spin-orbit coupling calculations.

\section{AUTHOR CONTRIBUTIONS}

A.M. and D.S.A. developed the idea, and A.M. performed the calculations. S.P. and S.R. $P$ provided the SQS structures. D.C. contributed to the DFT calculations. A.M. and D.S. A drafted the manuscript, which was edited by all authors. All authors collectively discussed the work and provided critical insights to the direction of the work.

\section{ADDITIONAL INFORMATION}

Supplementary information accompanies the paper on the npj Computational Materials website (https://doi.org/10.1038/s41524-018-0102-y).

Competing Interests: The authors declare no competing interests.

Publisher's note: Springer Nature remains neutral with regard to jurisdictional claims in published maps and institutional affiliations.

\section{REFERENCES}

1. Fultz, B. Vibrational thermodynamics of materials. Prog. Mater. Sci. 55, 247-352 (2010).

2. Wolverton, C. \& Zunger, A. Ni-Au: a testing ground for theories of phase stability. Comput. Mater. Sci. 8, 107-121 (1997).

3. Ozolins, V. \& Asta, M. Large vibrational effects upon calculated phase boundaries in Al-Sc. Phys. Rev. Lett. 86, 448-451 (2001).

4. Ozolins, V., Wolverton, C., \& Zunger, A. First-principles theory of vibrational effects on the phase stability of Cu-Au compounds and alloys. Phys. Rev 58, 10 (1998).

5. van de Walle, A. \& Ceder, G. The effect of lattice vibrations on substitutional alloy thermodynamics. Rev. Mod. Phys. 74, 11-45 (2002).

6. van de Walle, A., Ceder, G. \& Waghmare, U. W. First-principles computation of the vibrational entropy of ordered and disordered Ni3Al. Phys. Rev. Lett. 80, 4911-4914 (1998).

7. Jiang, C., Wolverton, C., Sofo, J., Chen, L.-Q. \& Liu, Z.-K. First-principles study of binary bcc alloys using special quasirandom structures. Phys. Rev. B 69, 214202 (2004).

8. Colinet, C., Eymery, J., Pasturel, A., Paxton, A.T. \& van Schilfgaarde, M. Firstprinciples phase stability study in the Au-Ni system. J. Phys. Condens. Matter 6, L47-L52 (1994).

9. Zaddach, A. J., Scattergood, R. O. \& Koch, C. C. Tensile properties of low-stacking fault energy high-entropy alloys. Mater. Sci. Eng. A 636, 373-378 (2015).

10. Bogdanoff, P. D. \& Fultz, B. Vibrational entropies of alloying and compound formation: experimental trends. Philos. Mag. Part B 79, 753-765 (1999).

11. Doak, J. W., Wolverton, C., \& Ozolins, V. Vibrational contributions to the phase stability of PbS-PbTe alloys. Phys. Rev. B 92, 174306 (2015).

12. Kessler, S. H., Abrecht, D. G., Clark, R. A. \& Schwantes, J. M. Vibrational contributions to phase stability in the Mo-Ru system. J. Alloy. Compd. 689, 969-976 (2016).

13. Muñoz, J. A. \& Fultz, B. Miscibility gap and phonon thermodynamics of Fe-Au alloys studied by inelastic neutron scattering and nuclear-resonant inelastic $\mathrm{x}$-ray scattering. AIP conference proceedings 1671, 020001 (2015).

14. Mantina, M., Wang, Y., Arroyave, R., Chen, L., Liu, Z., \& Wolverton, C. Firstprinciples calculation of self-diffusion coefficients. Phys. Rev. Lett. 100, 215901 (2008).

15. Benisek, A. \& Dachs, E. The vibrational and configurational entropy of disordering in Cu3Au. J. Alloy. Compd. 632, 585-590 (2015).

16. Yeh, J.-W. et al. Nanostructured high-entropy alloys with multiple principle elements: novel alloy design concepts and outcomes. Adv. Eng. Mater. 6, 299-303 (2004).

17. Cantor, B., Chang, I. T. H., Knight, P. \& Vincent, A. J. B. Microstructural development in equiatomic multicomponent alloys. Mater. Sci. Eng. A 375-377, 213-218 (2004).

18. Gludovatz, B., Hohenwarter, A., Catoor, D., Chang, E. H., George, E. P. \& Ritchie, R. O. A fracture-resistant high-entropy alloy for cryogenic applications. Science $\mathbf{3 4 5}$ 1153-1158 (2014).

19. Troparevsky, M. C., Morris, J. R., Kent, P., Lupini, A. \& Stocks, M. Criteria for predicting the formation of single-phase high-entropy alloys. Phys. Rev. X 5, 011041 (2015). (011041-011046).

20. Troparevsky, M. C., Morris, J. R., Daene, M., Wang, Y., Lupini, A. R. \& Stocks, G. M. Beyond atomic sizes and Hume-Rothery rules: understanding and predicting high-entropy alloys. JOM 67, 2350-2363 (2015).

21. Yeh, J.-W. Recent progress in high-entropy alloys. Ann. De. Chim. Sci. Des. Mater. 31, 633-648 (2006).

22. Otto, F., Yang, Y., Bei, H. \& George, E. P. Relative effects of enthalpy and entropy on the phase stability of equiatomic high-entropy alloys. Acta Mater. 61, 2628-2638 (2013).

23. Tomilin, I. A. \& Kaloshkin, S. D. 'High entropy alloys'-'semi-impossible' regular solid solutions? Mater. Sci. Technol. 31, 1231-1234 (2015).

24. Miracle, D. B. \& Senkov, O. N. A critical review of high entropy alloys and related concepts. Acta Mater. 122, 448-511 (2017).

25. Aidhy, D. S. \& Wolverton, C. First-principles prediction of phase stability and crystal structures in Li-Zn and Na-Zn mixed-metal borohydrides. Phys. Rev. B 83, 144111 (2011). 
26. Okamoto, H., Chakraborti, D. J., Laughlin, D. E. \& Massalski, T. B. The Au-Cu (gold-copper) system. J. Phase Equilibria 8, 454-474 (1987).

27. Swartzendruber, L. J., Itkin, V. P. \& Alcock, C. B. The Fe-Ni (iron-nickel) system. J. Phase Equilibria 13, 585-585 (1992).

28. Nash, P. \& Singleton, M. F. The Ni-Pt (nickel-platinum) system. Bull. Alloy Phase Diagr. 10, 258-262 (1989).

29. Shang, S. L., Wang, Y., Kim, D. E., Zacherl, C. L., Du, Y. \& Liu, Z. K. Structural, vibrational, and thermodynamic properties of ordered and disordered Ni1-xPtx alloys from first-principles calculations. Phys. Rev. B 83, 144204 (2011).

30. Okamoto, H. \& Massalski, T. B. The Ag-Au (silver-gold) system. Bull. Alloy Phase Diagr. 4, 30-38 (1983).

31. Franke, P. \& Neuschütz, D. Nb-Ta (Niobium - Tantalum). Thermodynamic Properties of Inorganic Materials. Binary Systems. Part 5: Binary Systems Supplement 1. Landolt-Börnstein - Group IV Physical Chemistry 19B5, 1-4. Springer (2007).

32. Calculated Pt-Ru phase diagram. Calculated Fe-Si phase diagram http://resource. npl.co.uk/mtdata/phdiagrams/ptru.htm Accessed 06 July 2017.

33. Collection of phase diagrams. FTlite-FACT light metal alloy phase diagrams (733) http://www.crct.polymtl.ca/fact/phase_diagram.php?file=Pt-Ru. jpg\&dir=SGTE2014Accessed 6 July 2017.

34. Tesfaye, F., Lindberg, D. \& Taskinen, P. The $\mathrm{Cu}-\mathrm{Ni}-\mathrm{S}$ system and its significance in metallurgical processes. EPD Congress 2016. 29-37 (2016).

35. Nash, A., \& Nash, P. The Ni-Rh (Nickel-Rhodium) system. Bulletin of Alloy Phase Diagrams 5, 403-405 (1984).

36. Karakaya, I. \& Thompson, W. T. The Ag-Pd (silver-palladium) system. Bull. Alloy Phase Diagr. 9, 237-243 (1988).

37. Calculated $\mathrm{Ag}-\mathrm{Pd}$ phase diagram. Calculated $\mathrm{Ag}-\mathrm{Pd}$ phase diagram. http:// resourcenplcouk/mtdata/phdiagrams/agpdhtm Accessed 6 July 2017.

38. Teeriniemi, J., Taskinen, P. \& Laasonen, K. First-principles investigation of the $\mathrm{Cu}-\mathrm{Ni}, \mathrm{Cu}-\mathrm{Pd}$, and Ni-Pd binary alloy systems. Intermetallics 57, 41-50 (2015).

39. Nash, A. \& Nash, P. The Ni-Pd (nickel-palladium) system. Bull. Alloy Phase Diagr. 5, 446-450 (1984).

40. Gao, M., Yeh, J. \& Liaw, P. High-entropy alloys; fundamentals and applications (pp. 351-357). Springer (2016).

41. Zaddach, A. J., Niu, C., Koch, C. C. \& Irving, D. L. Mechanical properties and stacking fault energies of NiFeCrCoMn high-entropy alloy. JOM 65, 1780-1789 (2013).
42. Lucas, M. S. Thermomagnetic analysis of FeCoCrxNi alloys: magnetic entropy of high-entropy alloys. J. Appl. Phys. 113(17), 17A923 (2013).

43. Kresse, G. \& Furthmuller, J. Efficiency of ab-initio total energy calculations for metels and semicoonductors using a plane-wave basis set. Comput. Mater. Sci. 6 , 15-50 (1996).

44. Perdew, J. P., Burke, K. \& Ernzerhof, M. Generalized gradient approximation made simple. Phys. Rev. Lett. 77, 3865-3868 (1996).

45. Monkhorst, H. J. \& Pack, J. D. Special points for Brillouin-zone integrations. Phys. Rev. B 13, 5188-5192 (1976).

46. Zunger, A., Wei, S. H., Ferreira, L. \& Bernard, J. Special quasirandom structures. Phys. Rev. Lett. 65, 353-356 (1990).

47. van de Walle, A. et al. Efficient stochastic generation of special quasirandom structures. Calphad 42, 13-18 (2013).

48. Togo, A. \& Tanaka, I. First principles phonon calculations in materials science. Scr. Mater. 108, 1-5 (2015).

49. Xie, W., Xiong, W., Marianetti, C. A., \& Morgan, D. Correlation and relativistic effects in $U$ metal and U-Zr alloy: validation of ab-initio approaches. Phys. Rev. B 88, 235128 (2013).

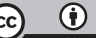

Open Access This article is licensed under a Creative Commons Attribution 4.0 International License, which permits use, sharing, adaptation, distribution and reproduction in any medium or format, as long as you give appropriate credit to the original author(s) and the source, provide a link to the Creative Commons license, and indicate if changes were made. The images or other third party material in this article are included in the article's Creative Commons license, unless indicated otherwise in a credit line to the material. If material is not included in the article's Creative Commons license and your intended use is not permitted by statutory regulation or exceeds the permitted use, you will need to obtain permission directly from the copyright holder. To view a copy of this license, visit http://creativecommons. org/licenses/by/4.0/.

(c) The Author(s) 2018 